\title{
Disseminated multiorgan MDR-TB resistant to virtually all first-line drugs
}

\author{
J. Sasse and D. Teichmann
}

ABSTRACT: The emergence of multidrug-resistant tuberculosis (MDR-TB) is a major global concern since, despite a complex treatment regime, it still remains a lethal threat.

A 21-yr-old male HIV-negative migrant from Burma presented with a disseminated tuberculosis affecting the lung, spleen, liver, mediastinal and abdominal lymph nodes. This particular strain of Mycobacterium tuberculosis proved to be resistant to all but one (pyrazinamide) of the first-line drugs, i.e. rifampicin, isoniazid and ethambutol, plus streptomycin, rifabutin and ofloxacin. On the mere account of its susceptibility concerning kanamycin it could not be labelled as extensively drug-resistant tuberculosis.

After 1 month of a standard first-line four-drug regimen and a subsequent 4 months of secondline treatment with amikacin, moxifloxacin, terizidone, protionamide, linezolid and pyrazinamide, sputum cultures eventually yielded constantly negative results. Likewise, the organ manifestations decreased significantly, so as to be virtually undetectable in computed tomography scans after $1 \mathrm{yr}$ of continuous treatment. A moderate pancytopenia reversed completely after dose adjustment of linezolid.

Disseminated tuberculosis manifestations without typical pulmonary cavernous lesions are likely to represent primary infection rather than reactivation. Even a multiorgan disseminated MDR-TB with an extensive resistance pattern (including fluoroquinolones) can be successfully treated with an individual second-line treatment and result in considerably few adverse events.

KEYWORDS: Clinical infectious diseases, drug resistance, extensively drug-resistant tuberculosis, multidrug-resistant tuberculosis, treatment, tuberculosis

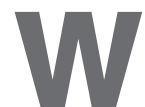
ith approximately 9 million new cases and 2 million deaths annually, tuberculosis (TB) remains one of the most devastating diseases worldwide [1].

The emergence of multidrug-resistant (MDR)-TB, i.e. $\mathrm{TB}$ in patients whose infecting isolates are resistant in vitro to at least isoniazid (INH) and rifampicin (RIF), poses a substantial therapeutic challenge.

MDR-TB with strains resistant to three or even four first-line drugs requires a complex and prolonged treatment regime comparable to that required in extensively drug-resistant (XDR)-TB, which, according to the definition revised by the US Centers for Disease Control and Prevention in collaboration with the World Health Organization (WHO) in 2006 [2], is defined as the occurrence of TB in persons whose Mycobacterium tuberculosis isolates are resistant to INH and RIF, plus resistant to any fluoroquinolone (FQ) and at least one of three injectable second-line drugs, i.e. amikacin, kanamycin or capreomycin. FQ and parenteral elements in second-line treatment, so the loss of one or both would inevitably weaken the regimen.

MDR-TB accounts for $2.1 \%$ and $4.5 \%$ of all TB cases reported annually in Germany and Burma, respectively [1]. However, the overall incidence of TB in these countries differs substantially, with rates of seven in 100,000 and 170 in 100,000, respectively. Owing to increases in international migration, MDR-TB should also be taken into consideration in low burden countries.

\section{CASE REPORT}

A 21-yr-old male patient from Burma living in Germany for 1 yr presented initially to a general practitioner with a 4-week history of retrosternal chest pain. Reflux oesophagitis was suspected and treated with ranitidine for 1 month without success. The patient was then transferred to a agents are deemed to be the most effective

\section{AFFILIATIONS}

Department of Infectious Diseases, Dresden-Neustadt Hospital, Dresden, Germany.

CORRESPONDENCE

J. Sasse

Kyffhaeuserstr. 32

D-01309 Dresden

Germany

E-mail: joerg.sasse@gmail.com

Received:

April 082009

Accepted after revision:

June 042009

PROVENANCE

Submitted article, peer reviewed. 
500-bed teaching hospital, where he mentioned a chronic cough with blood-tinged sputum.

Apart from a reduced state of nutrition $(170 \mathrm{~cm}, 49 \mathrm{~kg}$, body mass index $17 \mathrm{~kg} \cdot \mathrm{m}^{-2}$ ), the physical examination revealed no abnormality.

\section{LABORATORY FINDINGS}

Pathologically altered laboratory test results on admission included C-reactive protein $44.8 \mathrm{mg} \cdot \mathrm{L}^{-1}$, haemoglobin 7.82 $\mathrm{mmol} \cdot \mathrm{L}^{-1}$, white cell count $11,7000 \mu \mathrm{L}^{-1}$ and neutrophils $22 \%$. A HIV test, including HIV1/2 antibodies and p24 antigen, was negative.

A direct sputum smear on admission was positive for acid-fast bacilli (AFB); PCR confirmed M. tuberculosis complex.

\section{IMAGING}

Chest radiograph on admission showed extensive alveolar shadowing in the right middle lobe, bilateral hilar enlargement and right paratracheal lymphadenopathy (fig. 1).

Computed tomography (CT) 5 days after admission revealed bilaterally enlarged hilar lymph nodes and an alveolar opacification in the right middle lobe. Moreover, hypodense nodular structures in the lateral pole of the spleen (fig. 2) as well as in segment VIII of the liver were identified.

\section{MANAGEMENT AND TREATMENT OUTCOME}

Initially the patient was treated with a standard regime consisting of RIF $480 \mathrm{mg}$ q.d., INH $200 \mathrm{mg}$ q.d., pyrazinamide (PZA) 1,200 mg q.d. and ethambutol (EMB) 1,000 mg q.d.

Subsequent radiography and CT scans performed 4 weeks after treatment showed no significant improvement. Moreover,

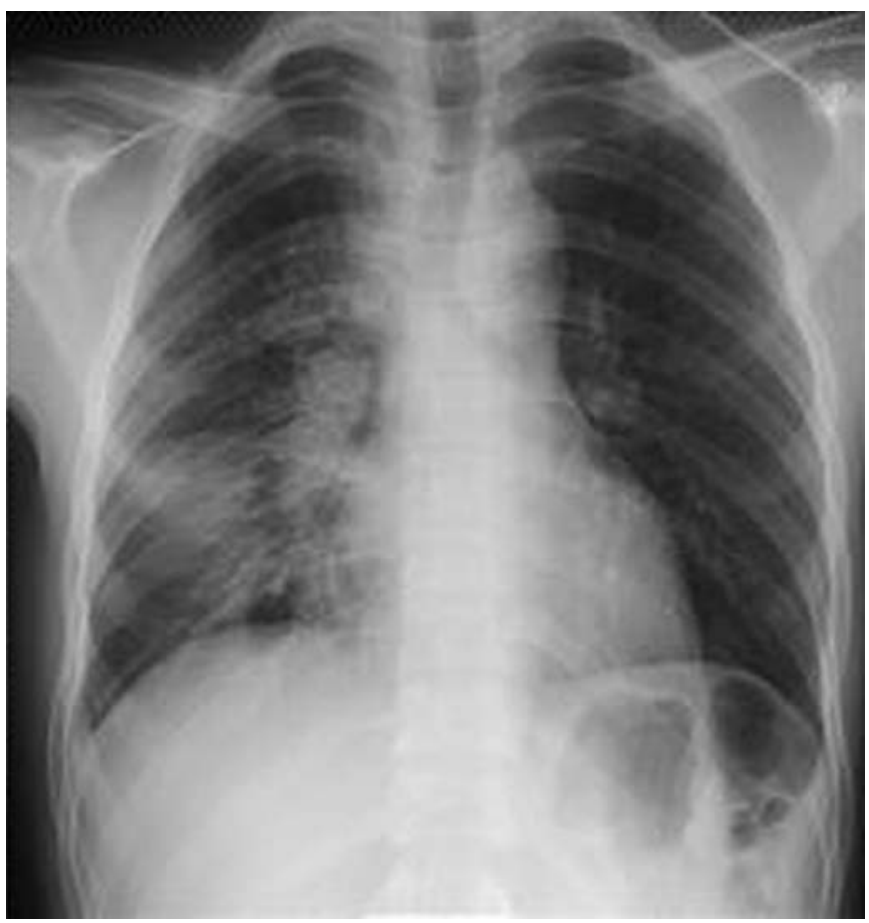

FIGURE 1. Chest radiograph on admission. all sputum smears collected within 7 weeks of treatment still displayed AFB and all of these cultures identified strains of $M$. tuberculosis.

Drug susceptibility testing (DST) proved in vitro resistance to the entire first-line drug regime, i.e. RIF, INH, PZA and EMB, plus streptomycin, rifabutin and ofloxacin.

According to the recommendations of current $\mathrm{WHO}$ guidelines [3] we decided to stop the previous regime and to start with amikacin 1,000 mg q.d., moxifloxacin $400 \mathrm{mg}$ q.d., terizidone $250 \mathrm{mg}$ t.i.d., protionamide $250 \mathrm{mg}$ t.i.d. and pyridoxine $50 \mathrm{mg}$ q.d. Due to the significant extent of resistance we also added linezolid $600 \mathrm{mg}$ b.i.d.

The National Reference Laboratory for mycobacteria in Borstel, Germany updated after a second DST that this strain was now sufficiently susceptible to PZA, leading to reintroduction of this drug. Furthermore, protionamide, amikacin, capreomycin, cycloserine/terzidone, moxifloxacin and linezolid yielded likewise results in vitro. Kanamycin was not tested at that time, because it is not licensed for systemic application in Germany. Eventually our patient's strain was found to be sensitive to kanamycin.

The patient tolerated this multidrug regime very well, apart from a slightly enhanced retrosternal burning pain caused by a chronic gastritis attributable to Helicobacter pylori, which resolved after an eradictaion regime.

A moderate decrease of all cell lines was fully reversible after the linezolid dosage was halved to $600 \mathrm{mg}$ q.d.

After 6 weeks of second-line treatment three consecutive early morning sputum samples were negative in AFB stains, yet it took another 3 months to achieve three consecutive negative cultures necessary to lift the strict airborne isolation precautions and eventually discharge the patient. He was subsequently seen regularly as an outpatient at our clinic. His sputum smears remained negative both on AFB stain and culture.

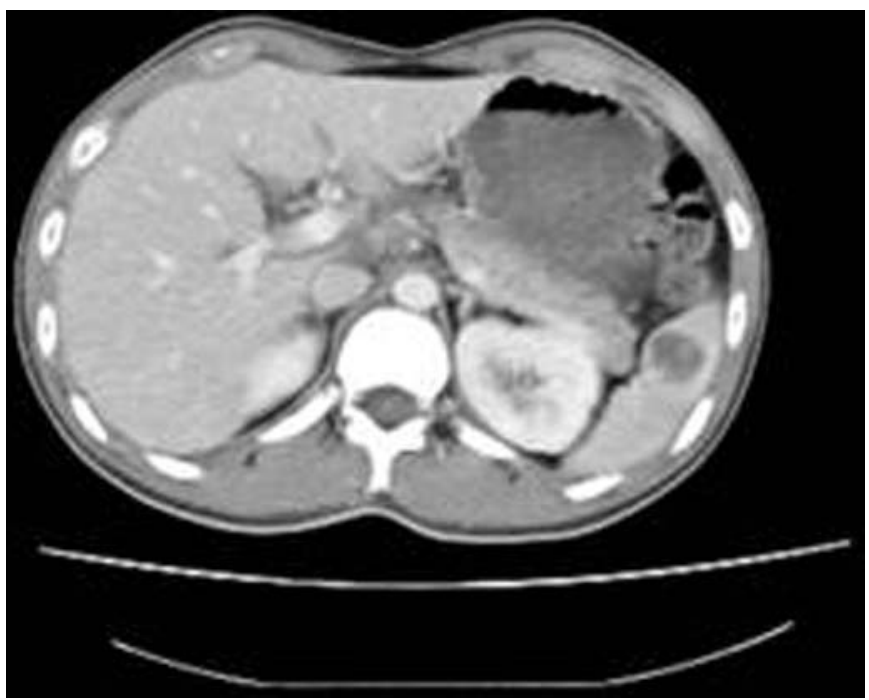

FIGURE 2. Computed tomography 5 days after admission. 


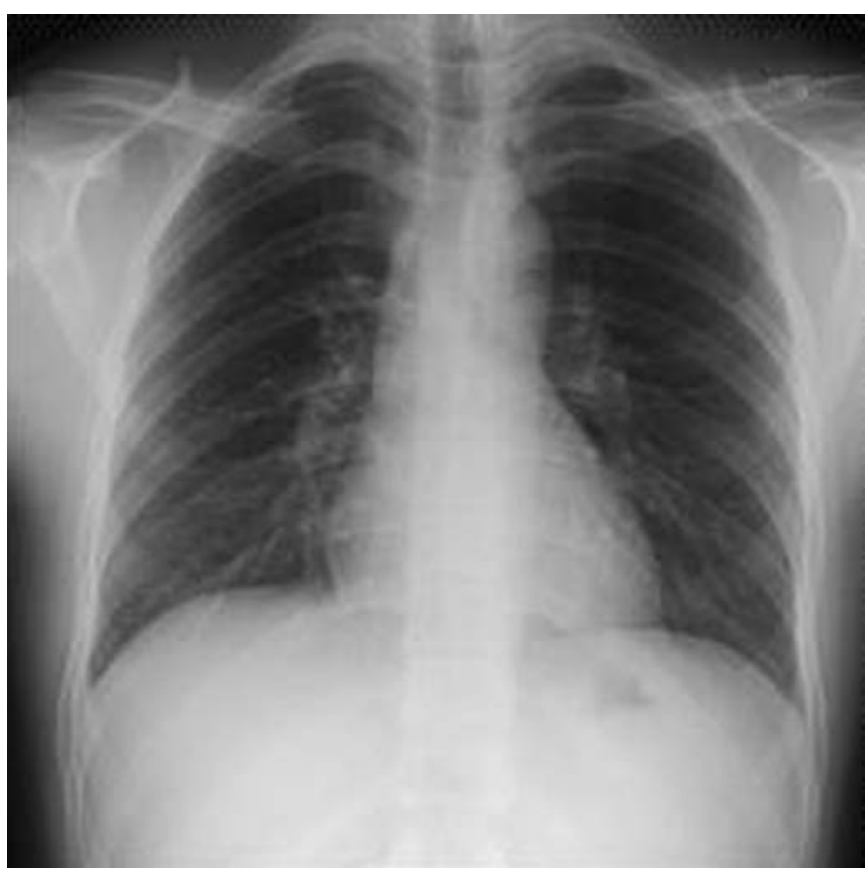

FIGURE 3. Chest radiograph after 9 months of second-line treatment.

The pulmonary lymphadenopathy and infiltrates decreased after 2 months and virtually disappeared after 9 months of second-line treatment (fig. 3). The abdominal TB correlates downsized to $<1 \mathrm{~cm}$ in diameter within the spleen, and disappeared entirely in the liver.

Contact tracing performed by both the Department of Hospital Epidemiology and Hygiene and the local health authorities detected no further cases of transmission after 22 months, but is required to cover a total of $2 \mathrm{yrs}$ according to WHO guidelines [3].

The continuation phase will continue for at least 18 months after sputum conversion and consists of moxifloxacin, PZA, terizidone and protionamide.

\section{DISCUSSION}

This case demonstrates common pitfalls in diagnosing $\mathrm{TB}$, i.e. the incorrect initial working diagnosis of an oesophagitis. However, TB should always be high on the list of differential diagnoses, and not only in patients who have spent a significant time in high-burden settings.

In a patient failing on treatment, drug resistance must be considered. The individual resistance pattern might feature an extensive pattern and offer only a few treatment options, even in non-XDR-TB. Hence, the DST should include all available second-line drugs to guide therapy. Despite a high overall mortality, MDR-TB, and even XDR-TB, remain treatable diseases as described in recent literature [4-7].

Patients with MDR-TB frequently have advanced disease associated with thick-walled cavities and chronic lung lesions that can be difficult for antimicrobial drugs to penetrate [8]. Our patient had unusual disseminated manifestations in the liver and spleen without typical cavernous lesions in the lung. This, in combination with lymphadenopathy, is likely to represent primary infection rather than reactivation (cavitating disease). Nevertheless, all TB manifestations decreased within the first 6 months of second-line therapy.

The choice of an appropriate medication had to meet both international guidelines and the extended resistance pattern of this MDR-TB strain, which was close to the criteria required to be labelled as XDR-TB. Our patient's strain featured resistance in vitro to RIF, INH, PZA and EMB, plus streptomycin, rifabutin and ofloxacin. Hence, if the strain had been resistant to kanamycin too, this disease would have had to be defined as XDR-TB.

An increase of resistance to $\mathrm{FQ}$ is of a particular concern because of its efficacy and tolerability [9, 10]. Although our patient's strain was resistant to ofloxacin, using moxifloxacin (according to the DST results) contributed to sputum conversion.

Our patient suffered from remarkably few side-effects, unlike previously suspected [11] in the course of this multidrug regime, which had even been enhanced by a transient $H$. pylori eradication regime.

A moderate pancytopaenia due to linezolid was entirely reversible after dose adjustment. It seems noteworthy that linezolid is actually licensed only for a duration of treatment no longer than 28 days, on account of potentially irreversible side-effects, such as peripheral neuropathy, which our patient did not develop after a total treatment duration of 184 days. Another substantial limitation for its application, especially in high-burden, low-resource settings, might be its high costs.

\section{STATEMENT OF INTEREST}

None declared.

\section{REFERENCES}

1 World Health Organization. Global Tuberculosis Control: Surveillance, Planning, Financing. Geneva, World Health Organization, 2008. Available from www.who.int/tb/publications/ global_report/2008/en/index.html

2 Centers for Disease Control and Prevention. Revised definition of extensively drug-resistant tuberculosis. Notice to readers. MMWR 2006; 55: 1176.

3 World Health Organization. Guidelines for the Programmatic Management of Drug-resistant Tuberculosis. Emergency Update 2008. Geneva, World Health Organization, 2008. Available from www.who.int/tb/publications/2008/programmatic_guidelines_ for_mdrtb/en/index.html

4 Mitnick CD, Shin SS, Seung KJ, et al. Comprehensive treatment of extensively drug-resistant tuberculosis. $N$ Engl J Med 2008; 359: 563-574.

5 Keshavjee S, Gelmanova IY, Farmer PE, et al. Treatment of extensively drug-resistant tuberculosis in Tomsk, Russia: a retrospective cohort study. Lancet 2008; 372: 1403-1409.

6 Sotgiu G, Ferrara G, Matteelli A, et al. Epidemiology and clinical management of XDR-TB: a systematic review by TBNET. Eur Respir J 2009; 33: 871-881.

7 Orenstein EW, Basu S, Shah NS, et al. Treatment outcomes among patients with multidrug-resistant tuberculosis: systematic review and meta-analysis. Lancet Infect Dis 2009; 9: 153-161.

8 Mukherjee JS, Rich ML, Socci AR, et al. Programmes and principles in treatment of multidrug-resistant tuberculosis. Lancet 2004; 363: $474-481$. 
9 Agrawal D, Udwadia ZF, Rodriguez C, et al. Increasing incidence of fluoroquinolone-resistant Mycobacterium tuberculosis in Mumbai, India. Int I Tuberc Lung Dis 2009; 13: 79-83.

10 Migliori GB, Lange C, Centis R, et al. Resistance to second-line injectables and treatment outcomes in multidrug-resistant and extensively drug-resistant tuberculosis cases. Eur Respir J 2008; 31: $1155-1159$.

11 Nathanson E, Gupta R, Huamani P, et al. Adverse events in the treatment of multidrug-resistant tuberculosis: results from the DOTS-Plus initiative. Int J Tuberc Lung Dis 2004; 8: 1382-1384. 